\title{
Dynamics of the Josephson Multi-Junction Systems with Junctions Characterized by Non-Sinusoidal Current - Phase Relationship
}

\author{
I.S. ABAL'OSheVA AND S.J. LeWANDOWSKI \\ Instytut Fizyki PAN, al. Lotników 32/46, 02-668 Warszawa, Poland \\ It is shown that the inclusion of junctions characterized by non- \\ -sinusoidal current - phase relationship in the systems composed of mul- \\ tiple Josephson junctions - results in the appearance of additional system \\ phase states. Numerical simulations and stability considerations confirm \\ that these phase states can be realized in practice. Moreover, spontaneous \\ formation of the grain boundary junctions in high- $T_{\mathrm{c}}$ superconductors with \\ non-trivial current-phase relations due to the $d$-wave symmetry of the or- \\ der parameter is probable. Switching between the phase states of multiple \\ grain boundary junction systems can lead to additional $1 / f$ noise in high- $T_{\mathrm{c}}$ \\ superconductors.
}

PACS numbers: $74.50 .+\mathrm{r}, 85.25 . \mathrm{Am}, 85.25 . \mathrm{Dq}$

\section{Introduction}

The interest in the properties of the Josephson multi-junction systems is to a large extent caused by the fact that granular superconductors, including the high- $T_{\mathrm{c}}$ materials, are usually modelled as arrays or loops of superconducting islands coupled by the Josephson junctions. Stationary theory developed for multi-junction interferometers (superconducting loops containing several Josephson junctions) [1] considers a system of junctions exhibiting the usual sinusoidal current-phase relationship (CPR) $I=I_{\mathrm{c}} \sin \varphi$. This theory predicts that the system can enter what is called a higher phase state characterized by phase difference across one or more junctions $\varphi_{n i}$ being restricted modulo $2 \pi$ to the interval $[\pi / 2,3 \pi / 2]$ (the basic state occupies the interval $[-\pi / 2, \pi / 2]$ ). By $\varphi_{n i}$ we denote 
phase difference across $i$-th junction in interferometer branch $n$. The necessary condition for the existence of an extremum of the free energy of the system is then

$$
\alpha_{1}+\frac{1}{\cos \tilde{\varphi}_{1}}=-a_{2}\left(\alpha_{2}+\frac{1}{\cos \tilde{\varphi}_{2}}\right)
$$

where $\alpha_{n}=\sum_{i=2}^{N_{n}} a_{n i} / \cos \varphi_{n i}+\beta_{L n}, a_{2}=I_{\mathrm{c} 1} / I_{\mathrm{c} 2}, a_{n i}=I_{\mathrm{c} n 1} / I_{\mathrm{c} n i}, N_{n}$ is the number of junctions in branch $n$, single indices 1 and 2 refer to the junctions with the smallest critical current in each branch, and $\beta_{L i}=2 \pi L_{i} I_{\mathrm{c} i} / \Phi_{0}$. Equation (1) describes the locus of the stationary points of the system $\left(\tilde{\varphi}_{1}, \tilde{\varphi}_{2}\right)$. Pairs $\left(\tilde{\varphi}_{1}, \tilde{\varphi}_{2}\right)$ correspond to points on one of the main interferometer characteristics dependence of system critical current $J_{\mathrm{c}}=I_{\mathrm{c} 1}+I_{\mathrm{c} 2}$ on external magnetic field $\Phi_{\mathrm{e}}$ [2], which is easy to observe experimentally. Predictions of the stationary theory were verified by investigating system stability in the frame of resistively shunted junction (RSJ) model and by measurements performed on niobium four-junction interferometers [3]. It was found that higher states of a system formed by junctions characterized by trivial CPR, due to $s$-wave symmetry of the order parameter, are unstable.

However, the results of phase-sensitive experiments on high- $T_{\mathrm{c}}$ superconductors $[4,5]$ are consistent with $d_{x^{2}-y^{2}}$-wave symmetry of the pair potentials. DC Josephson current between two superconductors with $d_{x^{2}-y^{2}}$-wave pairing symmetry is described by [6]:

$$
I(\varphi)=\sum_{k \geq 1} I_{k} \sin k \varphi
$$

The low-temperature behavior of the Josephson current depends on the angle formed between the crystalline axis of superconductor with $d_{x^{2}-y^{2}}$-wave pairing symmetry and the normal to the junction interface (see Fig. 1), as the sign of the pair potential depends on the direction of quasiparticle motion. As a consequence, conditions of preparation and geometrical layout, which can affect mutual orientation of junction electrodes and crystalline axes, determine the properties of such junctions.

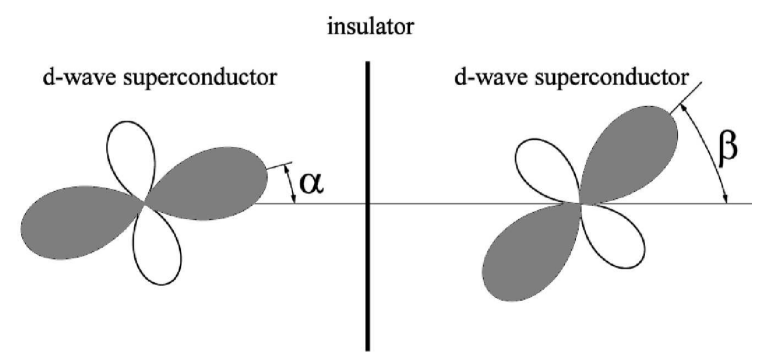

Fig. 1. Schematic representation of $d$-wave Josephson junction. 


\section{Josephson multi-junction systems on the base of $d$-wave superconductors}

In this paper, we discuss the possible appearance of additional system phase states in the case when multi-junction interferometer contains "nontrivial" junctions, i.e. junctions between $d$-wave superconductors. Let junction $n i$ be a non-trivial junction. To simplify the analysis we keep only two harmonics in Eq. (2):

$I_{\mathrm{c} n i}(\varphi)=I_{\mathrm{c} n i}^{*} \sin \varphi_{n i}+I_{\mathrm{c} n i}^{* *} \sin 2 \varphi_{n i}=I_{\mathrm{c} n i}^{*}\left(\sin \varphi_{n i}+b_{n i} \sin 2 \varphi_{n i}\right)$.

Then, looking for the extremum of the interferometer energy, we obtain the following modification of Eq. (1):

$$
\begin{aligned}
& \alpha_{1}+\frac{1}{\cos \tilde{\varphi}_{1}}=-a_{2}\left(\alpha_{2}+\frac{1}{\cos \tilde{\varphi}_{2}}\right), \\
& \sin \tilde{\varphi}_{n}=\frac{1}{a_{n i}}\left(\sin \varphi_{n i}+b_{n i} \sin 2 \varphi_{n i}\right),
\end{aligned}
$$

where $\alpha_{n}=\sum_{i=2}^{N_{n}} a_{n i} /\left(\cos \varphi_{n i}+2 b_{n i} \cos 2 \varphi_{n i}\right)+\beta_{L n}$. If $b=0$, this result reduces to Eq. (1), as it should be. The case of $b=0$ corresponds to zero angle between the crystalline $a$ axes of junction electrodes (assuming non-zero barrier potential [6]). Another extreme case is when the angle between the crystal axes is equal to $\pi / 4$. In such situation $I_{\mathrm{c}}^{*}$ (the lowest-order term) vanishes and Eq. (2) has a form close to $\sin 2 \varphi$. Other values of angles lead to different intermediate cases.

\section{The dynamics of the multi-junction interferometer containing "nontrivial" Josephson junctions}

The dynamics of the interferometer is described by the system of $N=$ $N_{1}+N_{2}$ equations

$$
\begin{gathered}
\beta_{\mathrm{c}} \ddot{\varphi}_{n i}+\dot{\varphi}_{n i}=(-1)^{n}\left[\sum_{k, m}(-1)^{k+1} \varphi_{k m} / \beta_{L}+2 \pi \phi_{\mathrm{e}} / \beta_{L}\right] \\
+i_{\mathrm{e}}\left(L-L_{n}\right) / L-\left(\sin \varphi_{n i}-b_{n i} \sin 2 \varphi_{n i}\right) / a_{n i}+i_{\mathrm{F} n i}
\end{gathered}
$$

where $\beta_{c}=2 \pi I_{\mathrm{c} 1} R_{12} C_{1} / \Phi_{0}, \beta_{L}=2 \pi I_{\mathrm{c} 1} L / \Phi_{0}, \phi_{\mathrm{e}}=\Phi_{\mathrm{e}} / \Phi_{0}, i_{\mathrm{e}}$ is the external current normalized to $I_{\mathrm{c} 1}, L_{n}$ denotes the inductance of the relevant interferometer branch, $L=L_{1}+L_{2}, i_{\mathrm{F} n i}$ is noise current flowing through the resistor $R_{n i}$ shunting the Josephson element in RSJ model.

By applying the standard Lyapunov method [7] to the system of Eqs. (5), one can show that for $\varphi_{n i} \in[-\pi / 2+2 m \pi, 3 \pi / 2+2 m \pi], m= \pm 1, \pm 2, \ldots$ (corresponding to the higher state) and $b_{n i}<0.5$ the system has not a stable solution, whereas for $b_{n i} \geq 0.5$ such solutions can be found.

Figure 2 illustrates the foregoing results for a three-junction interferometer with a non-trivial junction 12 . We show the dependence of critical current on 

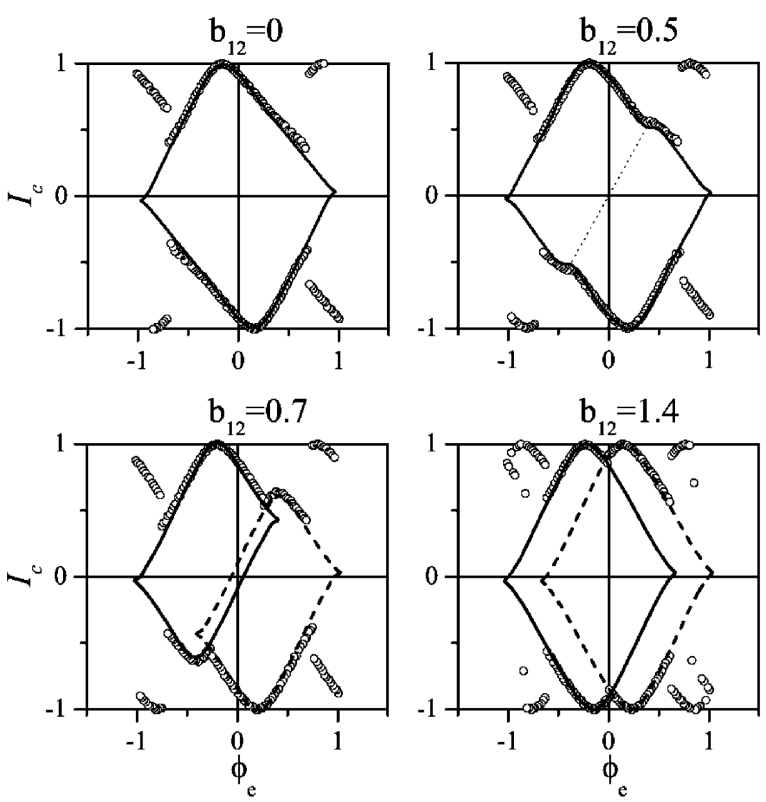

Fig. 2. Dependence of critical current of a three-junction interferometer on external magnetic field. The solid and dashed lines denote the critical currents obtained from Eq. (4). Circles are the critical currents obtained from numerical simulations of interferometer dynamics in the RSJ model.

external magnetic field obtained from numerical simulations of interferometer dynamics, and the $I_{\mathrm{c}}\left(\phi_{\mathrm{e}}\right)$ patterns determined by Eq. (4). One can see that only one (basic) interferometer phase state is occupied for $b_{12}<0.5$. When $b_{12}$ exceeds 0.5 , one can observe additional patterns corresponding to the higher state (dashed lines). In this case the system can occupy either the basic or the higher state and execute transitions between these states, depending on noise level (temperature).

\section{Energy consideration}

Appearance of additional higher states is easy to understand if we consider the expression for the energy of two connected in series non-trivial Josephson junctions

$$
U=-\left[i_{\mathrm{e}} \varphi_{1}+\cos \varphi_{1}-\frac{b_{1}}{2} \cos 2 \varphi_{1}+\frac{1}{a_{2}}\left(\cos \varphi_{2}-\frac{b_{2}}{2} \cos 2 \varphi_{2}\right)\right] .
$$

Due to the presence of $\cos 2 \varphi$ terms in (6), if either of the $b$ parameters is more or equal to 0.5 , four energy minima appear in the two-dimensional interval $[0,2 \pi] \times[0,2 \pi]$ of the phase space. Occupancy of one of the minima corresponds to one of the phase states of the considered system. 


\section{Conclusions}

To conclude, we investigated the dynamics of multi-junction systems of the Josephson junctions on the example of a three-junction interferometer. The numerical experiment based on solving the system of equations describing the interferometer dynamics and stability considerations following the Lyapunov method, show that higher phase states of the interferometer are allowed. Switching between the phase states of multiple grain-boundary junction systems can result in additional $1 / f$ noise in granular high critical temperature superconductors.

\section{Acknowledgments}

This work was supported by the State Committee for Scientific Research grant No. 2P 03B 04423.

\section{References}

[1] S.J. Lewandowski, Phys. Rev. B 43, 7776 (1991); S.J. Lewandowski, Acta Phys. Pol. A 80, 841 (1991); S.J. Lewandowski, Phys. Rev. B 45, 2319 (1992).

[2] A. Barone, G. Paterno, Physics and Applications of the Josephson Effect, Wiley, New York 1982.

[3] I.S. Abal'osheva, S.J. Lewandowski, Superconductor Sci. Technol. 15, 1220 (2002).

[4] D.J. van Harligen, Rev. Mod. Phys. 67, 515 (1995).

[5] C.C. Tsuei, J.R. Kirtley, Rev. Mod. Phys. 72, 969 (2000).

[6] Y. Tanaka, S. Kashiwaya, Phys. Rev. B 56, 892 (1997).

[7] A.B. Pippard, Response and Stability. An Introduction to the Physical Theory, Cambridge University Press, Cambridge 1985. 